\title{
Evolution of the Earth's Magnetosheath Turbulence: A Statistical Study Based on MMS Observations
}

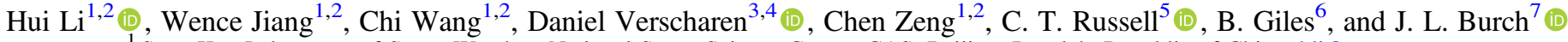 \\ ${ }^{1}$ State Key Laboratory of Space Weather, National Space Science Center, CAS, Beijing, People's Republic of China; hli@nssc.ac.cn, \\ jiangwence18@mails.ucas.edu.cn \\ ${ }^{2}$ University of Chinese Academy of Sciences, Beijing, People's Republic of China \\ ${ }^{3}$ Mullard Space Science Laboratory, University College London, Dorking RH5 6NT, UK \\ ${ }^{4}$ Space Science Center, University of New Hampshire, Durham, NH 03824, USA \\ ${ }^{5}$ University of California, Los Angeles, CA, USA \\ ${ }^{6}$ NASA Goddard Space Flight Center, Greenbelt, MD, USA \\ ${ }^{7}$ Southwest Research Institute, San Antonio, TX, USA \\ Received 2020 February 28; revised 2020 July 1; accepted 2020 July 13; published 2020 July 30
}

\begin{abstract}
Composed of shocked solar wind, the Earth's magnetosheath serves as a natural laboratory to study the transition of turbulence from low Alfvén Mach number, $M_{\mathrm{A}}$, to high $M_{\mathrm{A}}$. The simultaneous observations of magnetic field and plasma moments with unprecedented high temporal resolution provided by NASA's Magnetospheric Multiscale Mission (MMS) enable us to study the magnetosheath turbulence at both magnetohydrodynamics (MHD) and sub-ion scales. Based on 1841 burst-mode segments of MMS-1 from 2015 September to 2019 June, comprehensive patterns of the spatial evolution of magnetosheath turbulence are obtained: (1) from the subsolar region to the flanks, $M_{\mathrm{A}}$ increases from $<1$ to $>5$. At MHD scales, the spectral indices of the magnetic-field and velocity spectra present a positive and negative correlation with $M_{\mathrm{A}}$. However, no obvious correlations between the spectral indices and $M_{\mathrm{A}}$ are found at sub-ion scales. (2) From the bow shock to the magnetopause, the turbulent sonic Mach number, $M_{\text {turb }}$, generally decreases from $>0.4$ to $<0.1$. All spectra steepen at MHD scales and flatten at sub-ion scales, representing positive/negative correlations with $M_{\text {turb }}$. The break frequency increases by $0.1 \mathrm{~Hz}$ when approaching the magnetopause for the magnetic-field and velocity spectra, while it remains at $0.3 \mathrm{~Hz}$ for the density spectra. (3) In spite of minor differences, similar results are found for the quasi-parallel and quasi-perpendicular magnetosheath. In addition, such spatial evolution of magnetosheath turbulence is found to be independent of the upstream solar wind conditions, e.g., the averaged $Z$-component of the interplanetary magnetic field and solar wind speed.
\end{abstract}

Unified Astronomy Thesaurus concepts: Planetary magnetosphere (997); Interplanetary turbulence (830)

\section{Introduction}

Characterized by disordered fluctuations over a large range of scales, space plasma turbulence is of great importance in space physics due to its ubiquitous role in converting the fluctuation energy from large scales to small scales and eventually dissipating in collisionless magnetized plasmas (Schekochihin et al. 2009; Bruno \& Carbone 2013). To measure and study the multiscale nature of turbulence, one of the most common and insightful ways lies in the analysis of the power spectral density (PSD) of the turbulent fluctuations. From that perspective, the PSD of magnetic-field turbulence in the solar wind can generally be characterized by four distinguishable dynamical ranges of scales (e.g., Alexandrova et al. 2009; Sahraoui et al. 2009; Alexandrova et al. 2013; Bruno \& Carbone 2013; Goldstein et al. 2015; Huang et al. 2017, and the references therein): (1) a scaling of $\sim f^{-1}$ at energy-containing scales in Alfvénic fast solar wind and at larger scales in non-Alfvénic slow solar wind (Bruno et al. 2019); (2) a scaling of $f^{-5 / 3}$ (Kolmogorov 1941) or $f^{-3 / 2}$ (Iroshnikov 1964; Kraichnan 1965) in the inertial range or at magnetohydrodynamics (MHD) scales; (3) a scaling of $\sim f^{x}$ at sub-ion scales with a broader range of slopes, $x \in[-3.1,-2.3]$ with a narrow core distribution around $x=-2.8$; (4) even steeper scaling at electron scales.

The Earth's magnetosheath is a highly turbulent region bounded by the bow shock and the magnetopause. For the magnetic energy spectra in the magnetosheath, some similarities with those in the solar wind have been shown in previous studies (e.g., Alexandrova 2008; Riazantseva et al. 2017; Chhiber et al. 2018), for example, the existence of the $\sim f^{-1}$ scaling at large scales and the Kolmogorov spectral index $-5 / 3$ at MHD scales (Alexandrova et al. 2008; Huang et al. 2017; Chhiber et al. 2018), a broad distribution of slopes, $[-4,-2]$ with a peak near -2.8 , at sub-ion scales (Czaykowska et al. 2001; Alexandrova et al. 2008; Šafránková et al. 2013; Huang et al. 2014; Chen \& Boldyrev 2017; Zhu et al. 2019), and steeper spectra at electron scales (Matteini et al. 2017; Macek et al. 2018). However, due to the existence of multiple origins of waves and instabilities (Fairfield 1976; Omidi et al. 1994; Zimbardo et al. 2010), magnetosheath turbulence is more complicated than the turbulence in the solar wind. First, the bow shock and the magnetopause influence the magnetosheath turbulence properties (Gurnett et al. 1979; Rezeau \& Belmont 2001; Sahraoui et al. 2006; Rakhmanova et al. 2018b). Second, strong temperature anisotropy generally observed in the magnetosheath can generate various instabilities under different conditions, likely the Alfvén/ ion-cyclotron instability, the mirror-mode instability, the fast magnetosonic/whistler instability, and the fire-hose instability (e.g., Southwood \& Kivelson 1993; Quest \& Shapiro 1996; Gary et al. 1998; Hellinger \& Matsumoto 2000; Guicking et al. 2012; Kunz et al. 2014; Verscharen et al. 2016), which is verified by many studies (e.g., Anderson \& Fuselier 1993; 
Anderson et al. 1994; Schwartz et al. 1996; Czaykowska et al. 2001; Lucek et al. 2001; Sahraoui et al. 2006; Chen \& Boldyrev 2017; Teh \& Zenitani 2019; Vörös et al. 2019; Zhao et al. 2019a, 2019b). Third, other turbulent fluctuations related to local structures, e.g., current sheets, magnetic islands, and vortices, can further complicate the magnetosheath turbulence picture (e.g., Alexandrova 2008; Karimabadi et al. 2014; Huang et al. 2018). In addition, the turbulence properties are also different in the quasi-parallel and quasi-perpendicular magnetosheath (e.g., Czaykowska et al. 2001; Shevyrev et al. 2007; Macek et al. 2015; Breuillard et al. 2018; Rakhmanova et al. 2018a). Here, the quasi-parallel magnetosheath is defined as the magnetosheath behind a bow shock with quasi-parallel field geometry and likewise the quasi-perpendicular magnetosheath.

In the Earth's magnetosheath, the solar wind is subsonic after crossing the bow shock, although it returns to supersonic in the flanks. Thus, the magnetosheath is a good environment to investigate the evolution of turbulence from a small Alfvén Mach number, $M_{\mathrm{A}}$, to a large $M_{\mathrm{A}}$. However, the spatial evolution of magnetosheath turbulence has not been comprehensively addressed except for a few studies. Based on a previous case study, the intermittency of plasma turbulence increases in amplitude and anisotropy away from the bow shock (Yordanova et al. 2008), and the break frequency of ionflux spectra evolves to higher frequencies approaching the magnetopause (Rakhmanova et al. 2017). From a statistical perspective, Guicking et al. (2012) find a decay of wave intensity of low-frequency magnetic-field fluctuations along the streamlines in the Earth's magnetosheath, which quantitatively agrees with the theoretical concept of freely evolving/decaying turbulence; Huang et al. (2017) find that the $\sim f^{-1}$ spectral scaling of magnetic-field spectra at MHD scales is more likely located in the vicinity of the bow shock, while the Kolmogorov-like scaling at MHD scales located away from the bow shock toward the flank and magnetopause regions. Moreover, the spectral scaling at sub-ion scales flattens from the bow shock to the flank and the magnetopause. Similar results are obtained for the ion-flux spectra in the dayside magnetosheath (Rakhmanova et al. 2018b, 2018a).

To our knowledge, due to the limitations of the timeresolution of plasma instruments, the spatial evolution of turbulence spectral properties in the magnetosheath has not been addressed simultaneously for the magnetic field, ion density, and velocity fluctuations. NASA's Magnetospheric Multiscale (MMS) mission measures the magnetic field and plasma moments with unprecedented high time resolutions in the magnetosheath (Burch et al. 2016), which provides a unique opportunity to perform a statistical study on the turbulence evolution in the magnetosheath both at MHD scales and at sub-ion scales. Based on 1841 burst-mode segments of MMS-1 observations from 2015 September to 2019 June, we perform a comprehensive study of turbulent spectral indices in the dayside terrestrial magnetosheath from MHD scales to subion scales in this study. We focus on the evolution of the spectral indices and break frequencies in the magnetosheath from the subsolar region to the flanks, and from the bow shock vicinity to the magnetopause. In addition, we give a comparison of the quasi-perpendicular and quasi-parallel magnetosheath.

\section{Data Set and Methodology}

The high-temporal-resolution data provided by MMS mission enable us to probe magnetosheath fluctuations from MHD scales to sub-ion scales. The magnetic field is measured by the Fluxgate Magnetometer (FGM; Russell et al. 2016), and the particle moments are obtained by the Fast Plasma Investigation instrument (FPI; Pollock et al. 2016). For burstmode, the time resolutions of FGM and FPI (for ions) are $1 / 128 \mathrm{~s}$ and $0.15 \mathrm{~s}$, respectively. We conduct a statistical survey of spectral parameters of magnetosheath turbulence based on 1841 segments of burst-mode data from the MMS-1 spacecraft from 2015 September to 2019 June, with the magnetic-field, density, and velocity fluctuations simultaneously investigated both at MHD and at sub-ion scales.

To study the spectral parameters of magnetosheath turbulence, we first obtain the omnidirectional magnetic-field spectrum, the ion density spectrum, and the omnidirectional ion-velocity spectrum by performing a fast Fourier transform (FFT) on each burst-mode data segment. To ensure that the PSD is not contaminated by measurement uncertainties, we define a threshold for the signal-to-noise ratio $(\mathrm{S} / \mathrm{N}$ in $\mathrm{dB})$ as 10 (Sahraoui et al. 2013). For the FGM measurement in the magnetosheath, this constraint is generally satisfied because the $\mathrm{S} / \mathrm{N}$ is always greater than 10 for all the frequencies (Chhiber et al. 2018). For the particle moments, we calculate the $S / N$ from the FPI data products as

$$
\mathrm{S} / \mathrm{N}=10 \log _{10}\left[\frac{\delta X^{2}}{\delta X_{\text {sens }}^{2}}\right]
$$

where $\delta X$ and $\delta X_{\text {sens }}$ are the amplitude of the fluctuation and the level of the sensitivity floor at the spacecraft-frame frequency, $f_{\text {sc }}$.

For single-spacecraft measurements, Taylor's hypothesis is widely used to convert observed timescales to length scales, assuming fluctuations cross the spacecraft at a velocity faster than the dynamical timescale of interest. Taylor's hypothesis is usually satisfied in the solar wind, while in the magnetosheath it could be broken under two major conditions (Howes et al. 2014; Klein et al. 2014): (1) a slow flow with $V_{\mathrm{sw}} / V_{\mathrm{A}}<0.3$, where $V_{\mathrm{sw}}$ and $V_{\mathrm{A}}$ are the solar wind speed and Alfvén speed, respectively; (2) a dispersive regime in which whistler turbulence dominates. Based on our statistical survey, the slowest flow is found downstream of the bow shock nose as expected, and $V_{\mathrm{sw}} / V_{\mathrm{A}}$ is typically greater than 0.6. In addition, the dispersive whistler wave is generally beyond the scales discussed here. Thus, Taylor's hypothesis is satisfied in our study.

The PSDs of physical parameters in the magnetosheath usually steepen at kinetic scales, which correspond to the ion characteristic scales, e.g., $f_{\rho \mathrm{i}}$ and $f_{\text {di }}$ (Galtier 2006; Schekochihin et al. 2009; Chen et al. 2014; Šafránková et al. 2015). $f_{\rho \mathrm{i}}$, $=V_{\mathrm{sw}} / 2 \pi \rho_{\mathrm{i}}$, and $f_{\mathrm{di}},=V_{\mathrm{sw}} / 2 \pi d_{\mathrm{i}}$, are the Doppler-shifted frequencies corresponding to the proton gyroradius, $\rho_{\mathrm{i}}=$ $V_{\text {th } \perp} / \Omega_{\mathrm{i}}$, and the proton inertial length, $d_{\mathrm{i}}=V_{\mathrm{A}} / \Omega_{\mathrm{i}}$, respectively. Here, $V_{\text {th } \perp}=\sqrt{2 k_{\mathrm{B}} T_{\mathrm{i} \perp} / m_{\mathrm{i}}}$ is the perpendicular thermal speed, $\Omega_{\mathrm{i}}$ is the gyrofrequency, $k_{\mathrm{B}}$ is the Boltzmann constant, $T_{\mathrm{i}}$ is the proton temperature, $m_{\mathrm{i}}$ is the mass of a proton, and the subscript $\perp$ denotes perpendicular to the background magnetic field. For simplicity, we automatically determine the break frequency, $f_{\mathrm{b}}$, by minimizing the chi-square value of a two-stage power-law fitting procedure on the PSDs. Several spectral 
shapes at the scales of transition from MHD to kinetic regimes have been proposed, such as bumps or plateaus (Riazantseva et al. 2017; Rakhmanova et al. 2018a). To reduce interferences, we obtain the spectral indices at MHD scales $\left(\alpha_{1}\right)$ and at sub-ion scales $\left(\alpha_{2}\right)$ from the linear least-squares fitting of PSDs over two separated frequency bands. In order to statistically guarantee a reliable scale separation, we set the upper-limit frequency of the MHD range to $f_{\rho \mathrm{i}}$ (Bale et al. 2005; Chhiber et al. 2018), and the lower-limit frequency of sub-ion scales to $0.8 \mathrm{~Hz}$ (the mean of $f_{\mathrm{di}}$ plus one standard deviation). Thus, we choose the frequency band as $\left[0.02 \mathrm{~Hz}, f_{\rho \mathrm{i}}\right]$ for MHD scales. For magnetic-field spectra, the sub-ion range is set to be $[0.8,5.0] \mathrm{Hz}$, which is generally sufficient for fitting the spectral indices. For density and velocity spectra, the Nyquist frequency of the MMS FPI burst-mode data is $\sim 3.3 \mathrm{~Hz}$. However, high-frequency white noise (with a spectral slope of zero) is sometimes found in the PSD of density/velocity fluctuations, as shown in Figure 2 of Macek et al. (2018). To reduce the potential interference of noise, the upper frequency of the sub-ion range is set to be $2.0 \mathrm{~Hz}$ after a one-by-one case check. In our study, at least 80 data points are used for fitting the spectral indices of density/ velocity turbulence at sub-ion scales. In addition, to make the fitting results as reliable as possible, only those results satisfying the $99.5 \%$ confidence level are used. Nevertheless, further verifications with long enough intervals of the sub-ion range are needed based on simultaneous magnetic-field and plasma measurements with much higher time resolutions in the future.

Figure 1 shows an example of magnetosheath observations made by MMS-1 on 2018 March 4. MMS-1 enters into the magnetosheath (MSH) from the solar wind (SW) at around 00:43 UT and stays in the magnetosheath for more than 30 minutes. During 00:56:43-01:01:03 UT, the burst-mode data denoted by the light blue area are available. In this interval, MMS-1 is located in the subsolar region $\left(9.8,-2.7,-3.1 R_{\mathrm{E}}\right.$ in GSE coordinates) with a local time of about 11:00, and the angle between the bow shock normal and the interplanetary magnetic-field vector, $\Theta_{\mathrm{Bn}}$ is $46.2^{\circ}$. The plasma beta value, $\beta_{\mathrm{i}}$, is about 17.7, the ion temperature anisotropy is about 1.14 $\left(T_{\perp} \perp / T_{\mathrm{i} \|}\right.$, where $T_{\mathrm{i} \perp}$ and $T_{\mathrm{i} \|}$ are the ion temperature perpendicular to and parallel to the background magnetic field), and the the Alfvénic Mach number $\left(M_{\mathrm{A}}\right)$ is about 1.99. $\rho_{\mathrm{i}}$ and $d_{\mathrm{i}}$ are $84.4 \mathrm{~km}$ and $21.0 \mathrm{~km}$, respectively. Thus, $f_{\rho \mathrm{i}}$ and $f_{\mathrm{di}}$ are $0.19 \mathrm{~Hz}$ and $0.74 \mathrm{~Hz}$, respectively.

Based on the burst-mode data, we calculate the omnidirectional magnetic-field spectrum, the ion density spectrum, and the omnidirectional ion velocity and show them in Figures 1(h), (i), and (j), respectively. It is clear that the $S / N$ for density and velocity spectra are always greater than 10 . All three spectra present well-defined two-stage power laws at MHD scales and at sub-ion scales. For the magnetic-field spectrum shown in Figure $1 \mathrm{~h}$ ), the spectrum breaks at $\sim 0.25 \mathrm{~Hz}$, near $f_{\rho \mathrm{i}}$. At MHD scales, the spectrum scales as $\sim f^{-0.96}$, shallower than the $f^{-5 / 3}$ (Kolmogorov 1941) or $f^{-3 / 2}$ (Iroshnikov 1964; Kraichnan 1965) predictions from MHD, which is consistent with previous results (Czaykowska et al. 2001; Huang et al. 2017; Macek et al. 2018). The $\sim f^{-1}$ spectral scaling is typically interpreted as the result of the forcing (or energy injection), which indicates the presence of newly generated local fluctuations at the vicinity of the bow shock. The spectrum steepens to $f^{-2.87}$ at sub-ion scales, a slope consistent with many previous observations in the magnetosheath (Huang et al. 2014;
Macek et al. 2018). The formation of a Kolmogorov-like spectrum in the inertial range requires sufficient time (comparable to the nonlinear time) to develop (Chhiber et al. 2018). However, the transit time from the bow shock to the MMS-1 location is too short for an inertial range to fully develop, due to the close proximity to the bow shock. In the density spectrum shown in Figure 1 (i), the spectrum breaks at $\sim 0.5 \mathrm{~Hz}$. A significant spectral steepening from $f^{-1.88}$ at MHD scales to $f^{-3.23}$ at sub-ion scales is observed. As shown in Figure 1(j), a clear transition of the velocity spectrum from $f^{-1.94}$ at MHD scales to $f^{-2.97}$ at sub-ion scales is observed, with the $f_{\mathrm{b}} \sim$ $0.5 \mathrm{~Hz}$. At MHD scales, the $f^{-1.94}$ scaling agrees with the prediction $\left(f^{-2}\right)$ of compressible hydrodynamic Burgers turbulence (Gotoh \& Kraichnan 1993) and numerical results (Kim \& Ryu 2005). At sub-ion scales, the $f^{-2.97}$ scaling is also consistent with the results of Macek et al. (2018) in the magnetosheath and Šafránková et al. (2016) in the solar wind. Šafránková et al. (2016) show that velocity spectral indices, varying from -6 to -2 , are influenced by plasma beta, and the $f^{-3}$ scaling corresponds to a plasma beta of $\sim 0.01$ or $>10.0$. In our case, the plasma beta is 17.7 .

\section{Evolution of the Earth's Magnetosheath Turbulence}

\subsection{From the Subsolar Region to the Flanks}

Figure 2(a) gives the 2D distribution of $M_{\mathrm{A}}$ in the GSE-XY plane of the magnetosheath. The nominal positions of the Earth's magnetopause and the bow shock are estimated from the models proposed by Shue et al. (1997) and Chao et al. (2002), respectively. As expected, $M_{\mathrm{A}}$ increases gradually as the solar wind approaches the flanks. In the subsolar region, the mean $M_{\mathrm{A}}$ is about 1.6, while it grows to 5.2 in the flanks around $X=0 R_{\mathrm{E}}$. $M_{\mathrm{A}}$ in the central magnetosheath is generally greater than that in the vicinity of the bow shock and the magnetopause. From top to bottom, the middle three panels present the spectral indices of the magnetic-field, density and velocity spectra at MHD scales as a function of $M_{\mathrm{A}}$. The color of the data points indicates the local time (LT). Evidently, we find a well-organized pattern of $M_{\mathrm{A}}$ versus LT, in agreement with the $2 \mathrm{D}$ distribution of $M_{\mathrm{A}}$ shown in Figure 2 (a). For $M_{\mathrm{A}}>3$, most of the cases are located in the dawn and dusk flanks with $\mathrm{LT} \leqslant 8$ and $\mathrm{LT} \geqslant 16$. For $M_{\mathrm{A}}<2$, most of the cases are located in the subsolar region with $11 \leqslant \mathrm{LT}$ $\leqslant 13$. For the magnetic-field spectra at MHD scales, the spectral index is positively correlated with $M_{\mathrm{A}}$, with a correlation coefficient $(\mathrm{CC})=0.42$. Its mean value changes from -2 for $M_{\mathrm{A}}<0.8$ to $-3 / 2$ for $M_{\mathrm{A}}>5$. For the density spectra at MHD scales, the spectral index remains $\sim-7 / 3$ for different $M_{\mathrm{A}}$ with $\mathrm{CC}=0.05$, and no obvious relation between spectral index and $M_{\mathrm{A}}$ is found. For the velocity spectra at MHD scales, the spectral index is negatively correlated with $M_{\mathrm{A}}$, with $\mathrm{CC}=-0.33$. Its mean value changes from -2 for $M_{\mathrm{A}}<0.8$ to $-5 / 2$ for $M_{\mathrm{A}}>5$. The right three panels accordingly give the results for sub-ion scales. No clear relations between the spectral index and $M_{\mathrm{A}}$ are found for all the three types of spectra.

From the subsolar region to the flanks, the mean spectral indices of the magnetic field change from -2 for $M_{\mathrm{A}}<0.8$ to $-5 / 3$ or $-3 / 2$ for $M_{\mathrm{A}}>5$, implying that the magnetosheath turbulence evolves to be more fully developed in the flanks. Alexandrova (2008) and Alexandrova et al. (2008) suggest that the Kolmogorov-like magnetic-field spectrum at MHD scales can be observed in the flanks, where the transit time is long enough for turbulence development. Recently, 
(a)

(b)

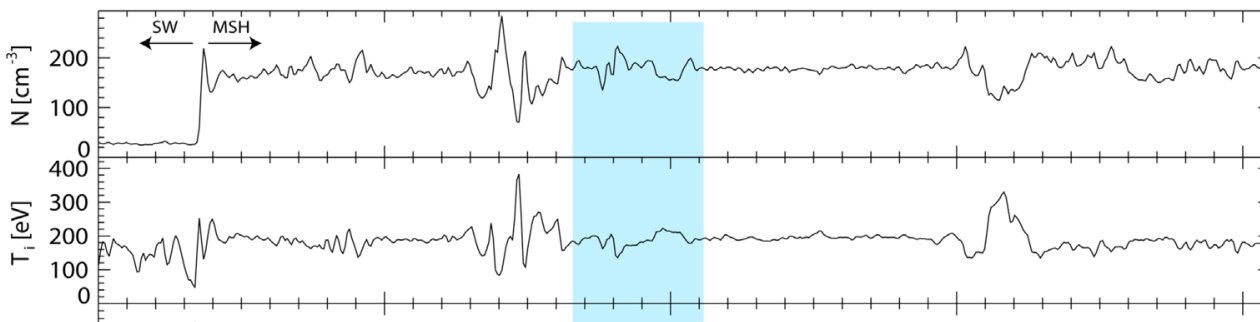

(c)

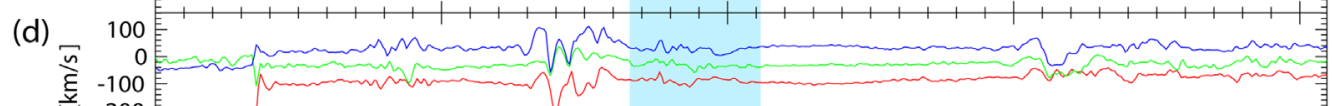
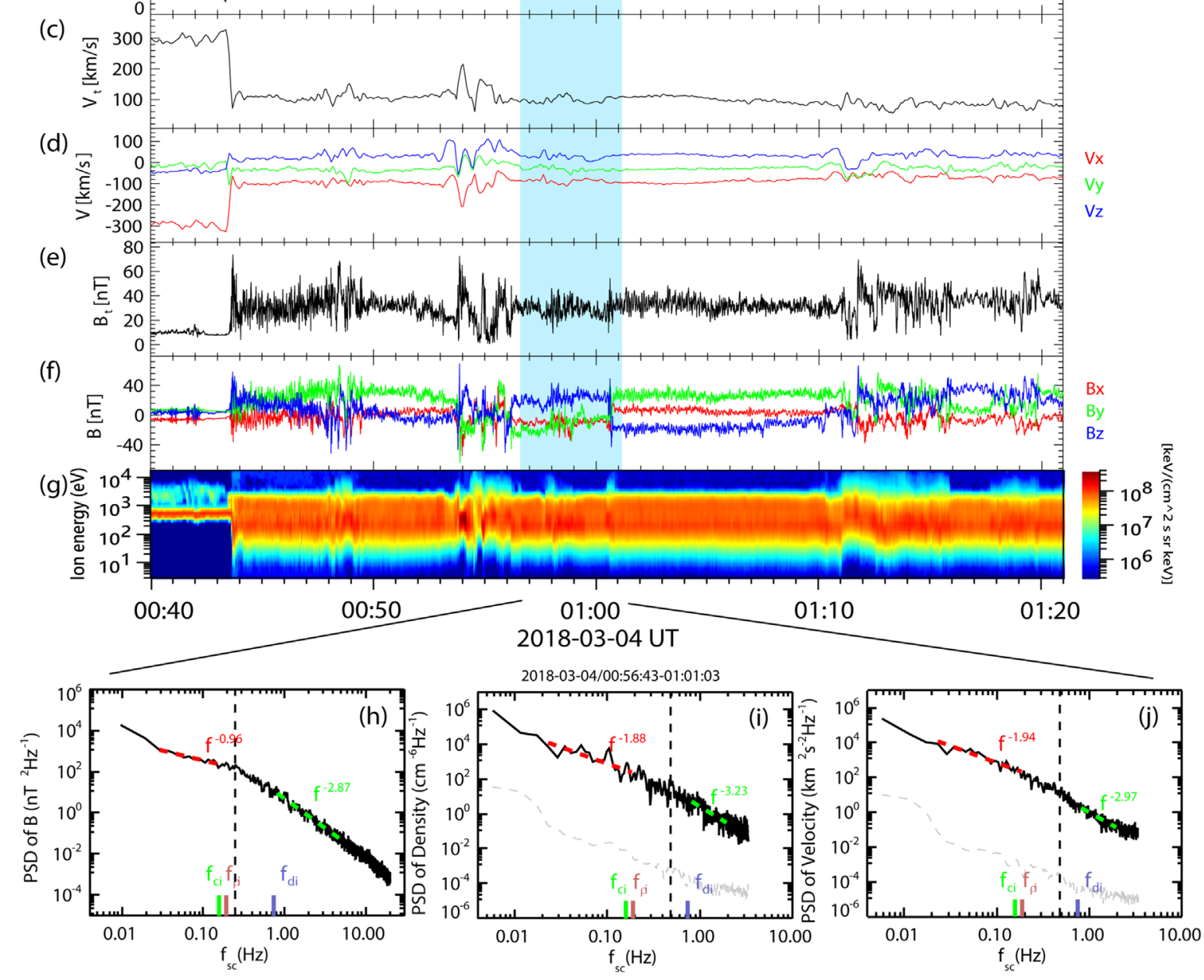

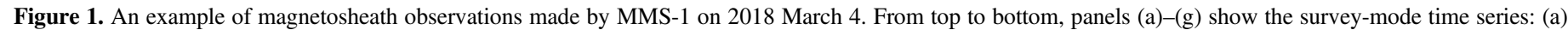

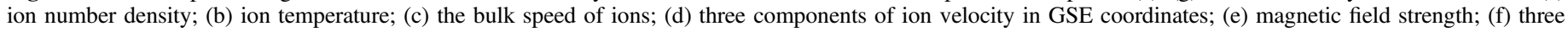

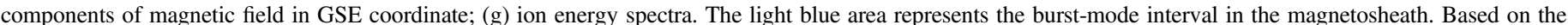

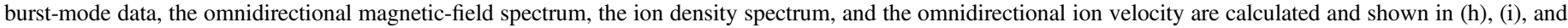

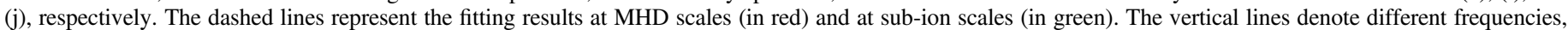

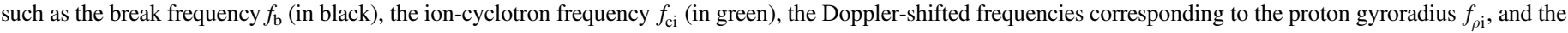
Doppler-shifted frequencies corresponding to the ion inertial length $f_{\mathrm{di}}$. The gray dashed lines in (i) and (j) represent the noise floor of the instrument.

Huang et al. (2017) also found that the $f^{-5 / 3}$ (Kolmogorovlike) magnetic-field spectra in the frequency range $\sim\left[10^{-4}, 10^{-1}\right] \mathrm{Hz}$ are only observed away from the bow shock to the flank and magnetopause, which is consist with our results. Here, we present a clear picture of this spatial evolution of MHD turbulence in the magnetosheath and, more importantly, the inverse dependences of magnetic-field and ion-velocity spectral indices on $M_{\mathrm{A}}$.

\subsection{From the Bow Shock to the Magnetopause}

To study the radial evolution of turbulent spectra inside the magnetosheath, the fractional distance between the spacecraft and the magnetopause $\left(D_{\text {frac }}\right)$ is calculated as proposed by
(Verigin et al. 2006)

$$
D_{\text {frac }}=\frac{r_{\mathrm{sc}}-r_{\mathrm{mp}}}{r_{\mathrm{bs}}-r_{\mathrm{mp}}}
$$

where $r_{\mathrm{sc}}, r_{\mathrm{mp}}$, and $r_{\mathrm{bs}}$ are the radial distances of the spacecraft, the Earth's magnetopause, and the bow shock away from the Earth center. $r_{\mathrm{sc}}$ is obtained from the MMS-1 observation directly. $r_{\mathrm{mp}}$ and $r_{\mathrm{bs}}$ can be derived from the empirical models (Shue et al. 1997; Chao et al. 2002) with given upstream solar wind conditions provided by the OMNI data. We also perform a comparison of the quasi-parallel and quasi-perpendicular magnetosheath here. Based on the bow shock model (Chao et al. 2002) and the OMNI data of upstream solar wind with a 

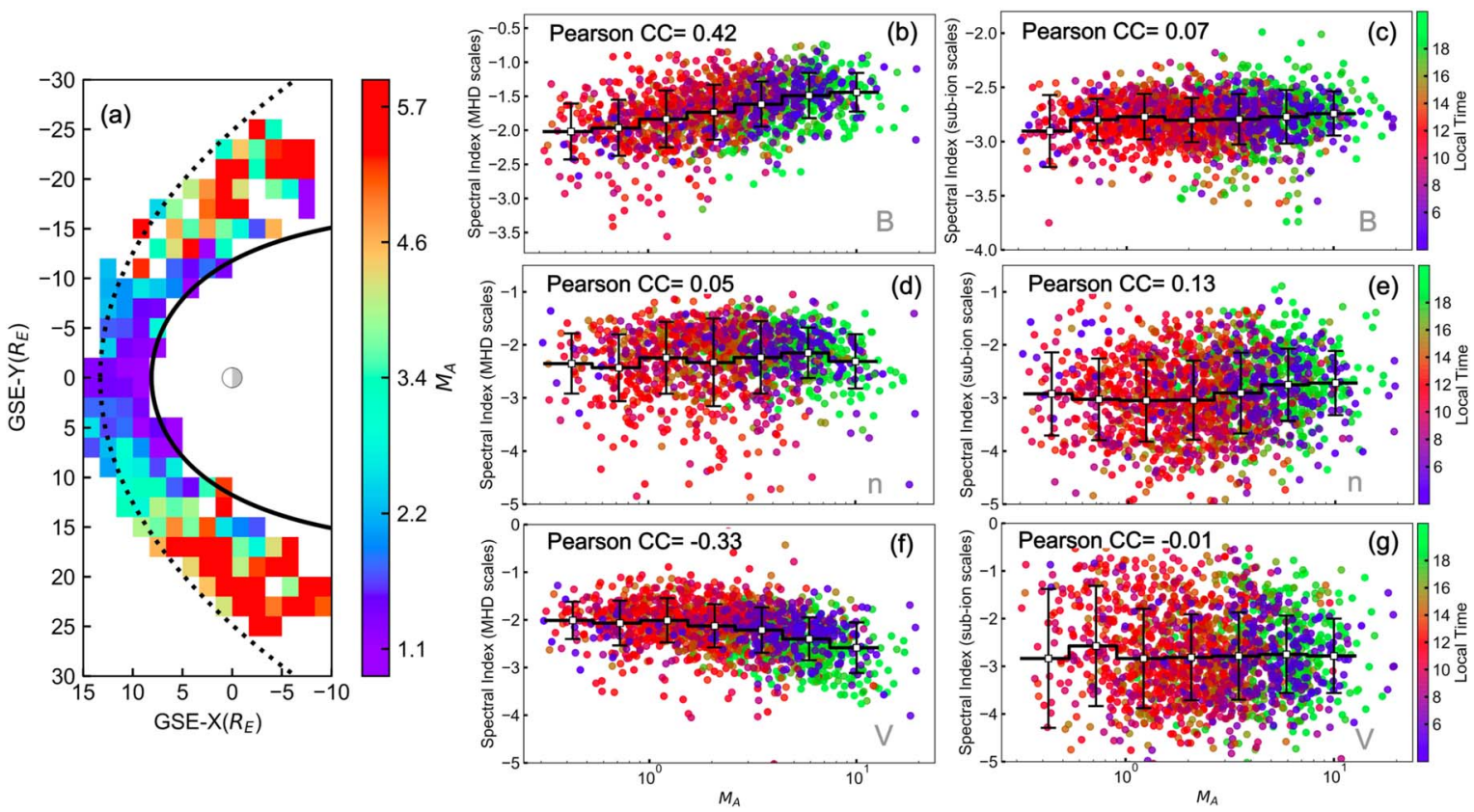

Figure 2. Evolution of magnetosheath turbulence from the subsolar region to the flanks. Panel (a) gives the 2D distribution of the Alfvén Mach number, $M_{\mathrm{A}}$, in the GSE-XY plane of the magnetosheath. The black solid and the black dotted curves represent the nominal position of the Earth's magnetopause and the bow shock estimated from the models proposed by Shue et al. (1997) and Chao et al. (2002), respectively. Panels (b), (d), and (f) present the magnetic-field spectral indices, the density spectral indices, and the velocity spectral indices at MHD scales as a function of $M_{\mathrm{A}}$. The results for sub-ion scales are accordingly given in panels (c), (e), and (g). The color of the data points indicates the local time. The black horizontal line represents the mean value for each bin, and the vertical line represents the standard deviation. CC represents the correlation coefficient between the spectral slope and $\log _{10} M_{\mathrm{A}}$.
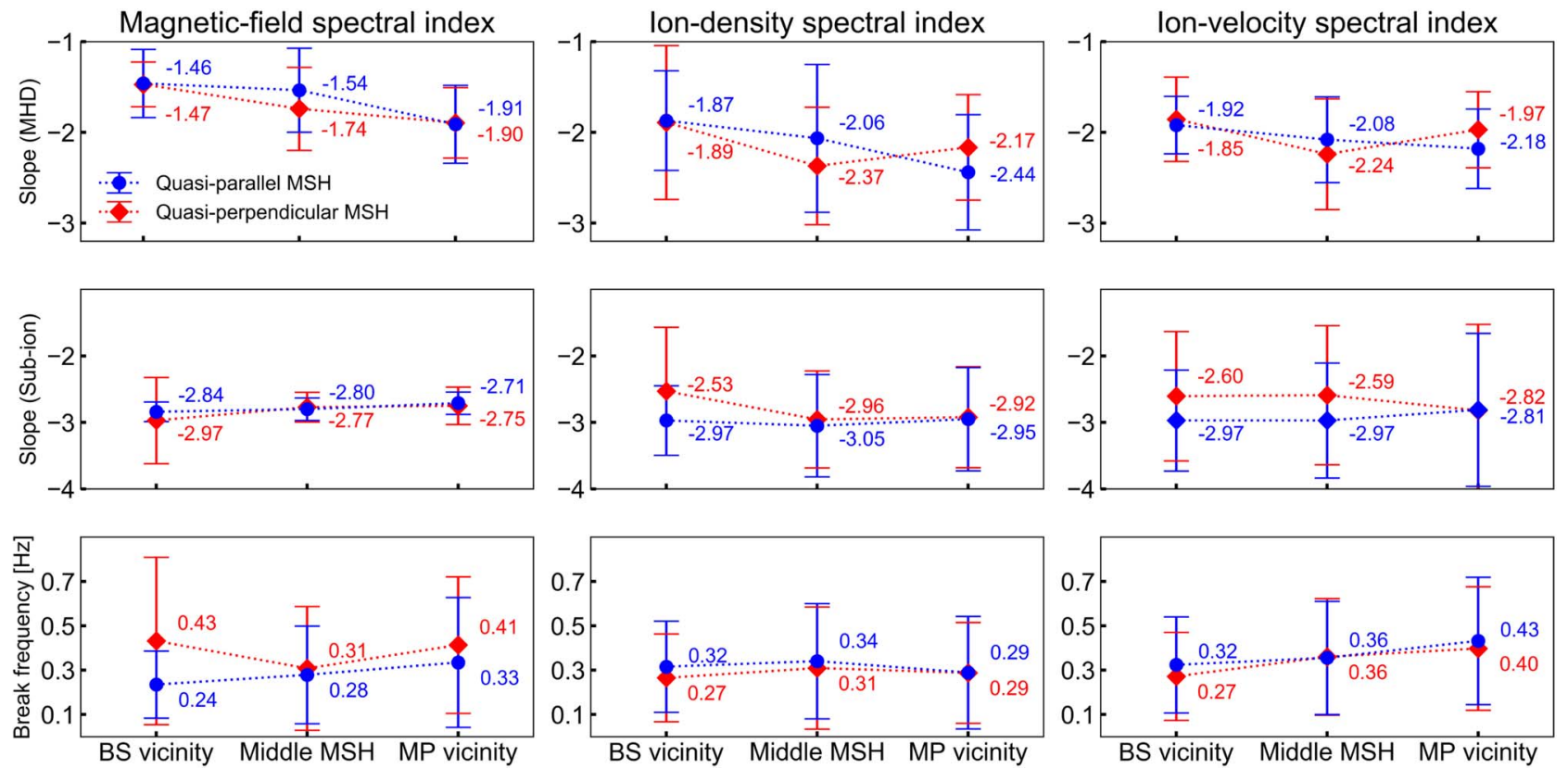

Figure 3. Evolution of magnetosheath turbulence from the bow shock to the magnetopause. In the top row, the three panels show the spectral slopes at MHD scales for magnetic-field spectra, density spectra, and velocity spectra, respectively. The results at sub-ion scales are given in the three panels in the middle row. The break frequency is given in the bottom three panels. The averaged values are denoted by blue circles (quasi-parallel magnetosheath) and red diamonds (quasi-perpendicular magnetosheath). The error bars indicate the standard deviation.

5 minute temporal resolution, we calculate the angle $\Theta_{\mathrm{Bn}}$ between the interplanetary magnetic field (IMF) and the normal of the bow shock surface corresponding to each data segment. Note that the location of MMS-1 is projected to the bow shock along the Earth's radial direction. To reduce the uncertainties, we define the quasi-parallel and quasi-perpendicular magnetosheath as $\Theta_{\mathrm{Bn}}<30^{\circ}$ and $\Theta_{\mathrm{Bn}}>60^{\circ}$, respectively.
We show the evolution of magnetosheath turbulence from the bow shock to the magnetopause in Figure 3. For the quasiparallel magnetosheath, the magnetic-field, density, and velocity spectra at MHD scales systematically and monotonically steepen from the bow shock to the magnetopause. The mean value of spectral index for magnetic-field spectra changes from -1.46 in the vicinity of the bow shock to -1.94 in the 
vicinity of the magnetopause. For density and velocity spectra, it changes from -1.87 to -2.44 and from -1.92 to -2.18 , respectively. At sub-ion scales, indistinct opposite trends of radial evolution are observed. For the magnetic-field spectra, the spectral slope changes from -2.84 in the vicinity of the bow shock to -2.71 in the vicinity of the magnetopause. For the density and velocity spectra, the spectral index changes from -2.97 to -2.95 and from -2.97 to -2.81 , respectively. Compared to the relatively large standard deviation, these differences are not significant. The break frequency for magnetic-field and velocity spectra increases when approaching the magnetopause, from 0.24 to $0.33 \mathrm{~Hz}$ and from 0.32 to $0.43 \mathrm{~Hz}$, respectively. For the density spectra, it remains around $0.3 \mathrm{~Hz}$. For the quasi-perpendicular magnetosheath, the results are overall similar, except for two differences: (1) for the density and velocity spectra, the steepest spectra at MHD scales occur in the central magnetosheath but not at the magnetopause, and the spectral slope in the bow shock vicinity is flatter than that at the magnetopause; (2) for the magnetic-field spectra, the break frequency is overall greater than that in the quasi-parallel magnetosheath. The lowest value, $0.31 \mathrm{~Hz}$, occurs in the central magnetosheath. In the vicinity of the bow shock and the magnetopause, no clear difference is found, around $0.41 \mathrm{~Hz}$.

At MHD scales, the magnetic-field, density, and velocity spectra generally steepen as the spacecraft approaches the magnetopause, especially for the quasi-parallel magnetosheath. Similar findings are reported for the magnetic-field spectra (Czaykowska et al. 2001; Sahraoui et al. 2006; Shevyrev et al. 2006) and the ion-flux spectra (Shevyrev et al. 2006), but not for the ion-density and velocity spectra. Various wave modes/ instabilities introduced immediately after the bow shock (e.g., Schwartz et al. 1996) may be responsible for these phenomena. Rakhmanova et al. (2018a) show that "bump"-type ion-flux spectra (i.e., peaked at $0.25-0.35 \mathrm{~Hz}$ ) are more likely observed near the bow shock rather than near the magnetopause, which leads to spectral flattening at MHD scales and spectral steepening at sub-ion scales. Sahraoui et al. (2006) argue that mirror-mode structures are more likely responsible for the "bumps" in magnetic field and plasma spectra with the peak at $\sim 0.1 \mathrm{~Hz}$.

At sub-ion scales, the magnetic-field spectra flatten from the bow shock to the magnetopause, which is consistent with the result of Rezeau et al. (1999). The average spectral index is -2.79 in the quasi-parallel magnetosheath and -2.78 in the quasi-perpendicular magnetosheath, respectively, which coincides with the result of a previous case study (Breuillard et al. 2018) and agrees with the prediction of kinetic Alfvén waves and whistler turbulence models. Rakhmanova et al. (2018a) show that the kinetic-scale ion-flux spectra have steeper indices in the bow shock vicinity than near the magnetopause, from -3.2 to -2.8 in the quasi-perpendicular and from -3.4 to -3.0 in the quasi-parallel magnetosheath with an averaged standard deviation of $0.4 \sim 0.5$. However, no significant differences are found from the bow shock to the magnetopause considering the large standard deviations in our study. One possibility is that Rakhmanova et al. (2018a) focus on the ion flux, while the density and velocity are investigated separately in this study. Besides, the different numbers of cases potentially contribute as well. Compared to Rakhmanova et al. (2018a), fewer cases (53 versus 174) in the vicinity of the bow shock and fewer cases (222 versus 237 ) in the vicinity of the magnetopause are used here. Moreover, Rakhmanova et al. (2017) show that the break frequency of ion-flux spectra increases from $0.25 \mathrm{~Hz}$ in the bow shock vicinity to $0.4 \mathrm{~Hz}$ in the magnetopause vicinity, which is similar to our result of velocity spectra, from about 0.3 to about $0.4 \mathrm{~Hz}$. Note that these values are much less than $0.6-0.8 \mathrm{~Hz}$ for ion-flux spectra as shown by Rakhmanova et al. (2018a). This discrepancy may result from the use of different physical parameters (velocity versus ion flux) and the effects of possible complex spectral shapes (e.g., bump or plateau found by Riazantseva et al. 2017; Rakhmanova et al. 2018a) when determining the spectral break.

Figure 4(a) gives the 2D distribution of the turbulent sonic Mach number, $M_{\text {turb }}=\delta V / C_{\mathrm{s}}$ (where $\delta V$ is the velocity fluctuation and $C_{\mathrm{s}}$ is the sound speed), in the GSE-XY plane of the magnetosheath. A large $M_{\text {turb }}$ implies a strongly compressed state and the existence of discrete density structures (Kritsuk et al. 2007). We find that $M_{\text {turb }}$ is well organized from the bow shock to the magnetopause. Large $M_{\text {turb }}(>0.4)$ are more likely to occur in the vicinity of the bow shock, and generally decrease to $<0.1$ closer to the magnetopause. From top to bottom, the middle three panels present the spectral indices of the magnetic-field, density, and velocity spectra at MHD scales as a function of $M_{\text {turb }}$. The color of the data points indicates the local time (LT). The spectral indices at MHD scales are all positively correlated with $M_{\text {turb }}$, with $\mathrm{CC}=0.24$ and 0.46 , and 0.34 , respectively. The mean spectral slops of magnetic-field, density and velocity spectra increase from $-1.9,-2.8,-2.6$ for $M_{\text {turb }}<0.08$ to -1.6 , $-1.8,-2.0$ for $M_{\text {turb }}>0.6$, respectively. The right three panels accordingly give the results for sub-ion scales. Compared to MHD scales, we find negative correlations between the spectral indices and $M_{\text {turb }}$ at sub-ion scales, with CC $=-0.20,-0.21$, and -0.37 for magnetic-field, density, and velocity spectra, respectively. These are consistent with the results shown in Figure 3, especially in the quasi-parallel magnetosheath.

As shown in Figure 1(h) and Figure 4(b), there exist some $f^{-1}$-like magnetic-field spectra at MHD scales. Hadid et al. (2015) and Huang et al. (2017) suggest that the interaction of solar wind and planetary bow shocks may be responsible for the formation of $f^{-1}$-like magnetic-field spectra in the magnetosheath. Another possible mechanism is the saturation of large-amplitude fluctuations (Matteini et al. 2018; Bruno et al. 2019). The steepening of magnetic-field spectral slope at MHD scales from the bow shock to the magnetopause is consistent with the picture that the turbulence downstream of the bow shock undergoes energy injection processes induced by, e.g., ion beams (Lucek et al. 2005), forming shallower magnetic-field spectra at MHD scales, and then evolves to more fully developed turbulence near the magnetopause. Early observations suggest that compressive turbulence in fast solar wind produces flat density spectra (Marsch \& Tu 1990) and show a good correlation between density fluctuations and sonic Mach number (Klein et al. 1993). In the context of compressive MHD turbulence, small high-density compressed regions associated with slow and fast modes dominate the flattened spectrum (Lithwick \& Goldreich 2001). In the supersonic regime, numerical simulations of hydrodynamic and MHD turbulence revealed that the density spectrum in the inertial range flattens when the rms sonic Mach number increases, while the velocity spectrum changes in the opposite sense (Kowal \& Lazarian 2007; Kritsuk et al. 2007). Banerjee \& Galtier (2013) attribute those results to the variability of the 

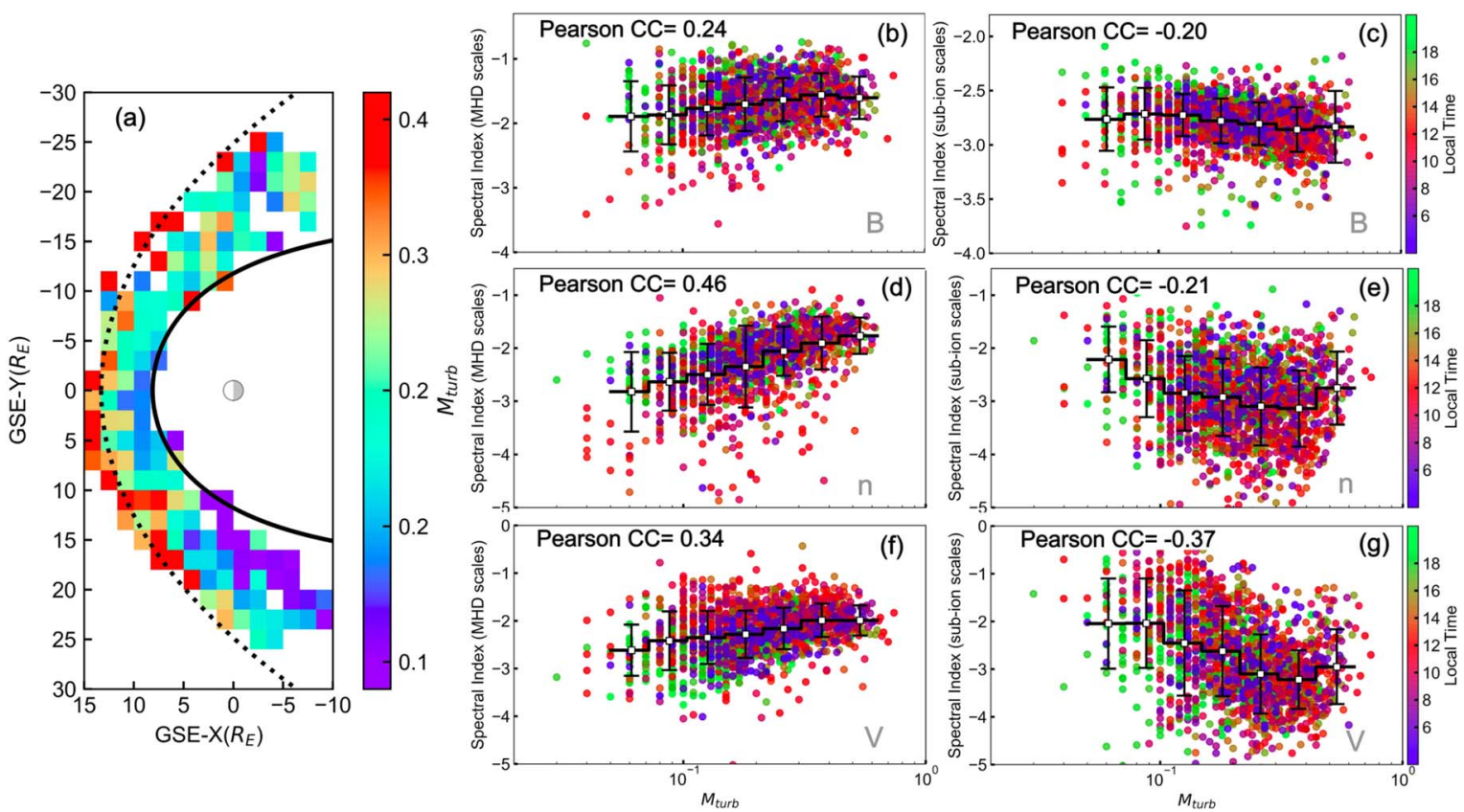

Figure 4. Evolution of magnetosheath turbulence from the bow shock to the magnetopause. Panel (a) gives the 2D distribution of the turbulent sonic Mach number, $M_{\text {turb }}$, in the GSE-XY plane of the magnetosheath. The black solid and the black dotted curves represent the nominal position of the Earth's magnetopause and the bow shock estimated from the models proposed by Shue et al. (1997) and Chao et al. (2002), respectively. Panels (b), (d), and (f) present the magnetic-field spectral indices, the density spectral indices, and the velocity spectral indices at MHD scales as a function of $M_{\text {turb }}$. The results for sub-ion scales are accordingly given in panels (c), (e), and (g). The color of the data points indicates the local time. The black horizontal line represents the mean value for each bin, and the vertical line represents the standard deviation. CC represents the correlation coefficient between the spectral slope and $\log _{10} M_{\text {turb }}$.

energy cascade rate due to compressibility. As shown in Figure 4(d), the density spectra at MHD scales flatten with increasing $M_{\text {turb }}$, which is consistent with previous numerical and experimental studies in the supersonic regime (Kritsuk et al. 2007; Kowal et al. 2007; Kowal \& Lazarian 2007; Konstandin et al. 2016; White et al. 2019). However, velocity spectral indices at MHD scales also flatten with the increasing of $M_{\mathrm{turb}}$, in contrast to the results in supersonic turbulence (Kowal \& Lazarian 2007; Kritsuk et al. 2007). Note that $M_{\text {turb }}$ is always less than 1 in the magnetosheath.

\subsection{Dependence on Upstream Solar Wind Conditions}

It is believed that the upstream solar wind conditions, e.g., the angle $\Theta_{\mathrm{Bn}}$, flow speed $V_{\mathrm{p}}$, and $z$-component of the IMF $B_{\mathrm{z}}$, modify the structure, dynamics, and dissipation processes in the magnetosheath (Dimmock et al. 2014). To investigate the influence of upstream solar wind conditions, represented by the averaged $V_{\mathrm{p}}$ and IMF $B_{\mathrm{z}}$, on the spatial evolution of magnetosheath turbulence, we compare the results of IMF $B_{\mathrm{z}}>0.5 \mathrm{nT}$ versus IMF $B_{\mathrm{z}}<-0.5 \mathrm{nT}$ and $V_{\mathrm{p}}>400 \mathrm{~km} \mathrm{~s}^{-1}$ versus $V_{\mathrm{p}}<400 \mathrm{~km} \mathrm{~s}^{-1}$ in Figure 5. In general, the profiles under different upstream conditions are almost identical. No systematical differences of the spectral indices as a function of $M_{\mathrm{A}}$ or $M_{\text {turb }}$ are found. In addition, no significant discrepancies of the correlation coefficients between three spectral indices and $M_{\mathrm{A}}$ or $M_{\text {turb }}$ are found either. These results indicate that the spatial evolution of magnetosheath turbulence is largely independent of the upstream solar wind conditions. The relative amplitudes of the downstream fluctuations, e.g., $\delta n / n_{0}$ and $\delta B / B_{0}$, are found to generally increase with the upstream solar wind speed. However, no obvious correlations between the spectral indices and $V_{\mathrm{p}}$ or IMF $B_{\mathrm{z}}$ are found. These two results are not shown here.
By studying the cross-correlations between upstream solar wind fluctuations and magnetosheath fluctuations, Gutynska et al. (2012) argue that low-frequency $\left(10^{-4}-10^{-2} \mathrm{~Hz}\right)$ fluctuations in the magnetosheath are generally from the solar wind, while high-frequency (up to $10^{-1} \mathrm{~Hz}$ ) fluctuations are generated locally in the magnetosheath. Rakhmanova et al. (2015) further confirm these results and suggest that the modification of solar wind structures by the bow shock and magnetosheath does not depend on $\Theta_{\mathrm{Bn}}$. In this study, we focus on the turbulence in a high-frequency range $(0.02-2.0 / 5.0 \mathrm{~Hz})$ that is more likely generated locally in the magnetosheath instead of being propagated from the upstream solar wind. Our results are limited to some extent since we use the averaged state of upstream solar wind assuming a steady upstream solar wind, but not a cross-correlation analysis of upstream and downstream observations. To what extent the penetrating small structures, such as plasma jets, especially behind a quasiparallel shock (Hietala et al. 2009), could influence the magnetosheath turbulence properties needs further investigation in the future.

\section{Summary}

The Earth's magnetosheath is a highly turbulent region bounded by the bow shock and the magnetopause, within which the solar wind reduces from supersonic to subsonic flow speeds after crossing the bow shock, and returns to a supersonic flow in the flanks. Thus, the magnetosheath provides a good natural laboratory to investigate the spatial evolution of space plasma turbulence from a small Alfvén Mach number, $M_{\mathrm{A}}$, to a large $M_{\mathrm{A}}$. By means of simultaneous observations of magnetic field and plasma moments with unprecedented high time resolution provided by the MMS mission, using 1841 burstmode segments of MMS-1 from 2015 September to 2019 June, 

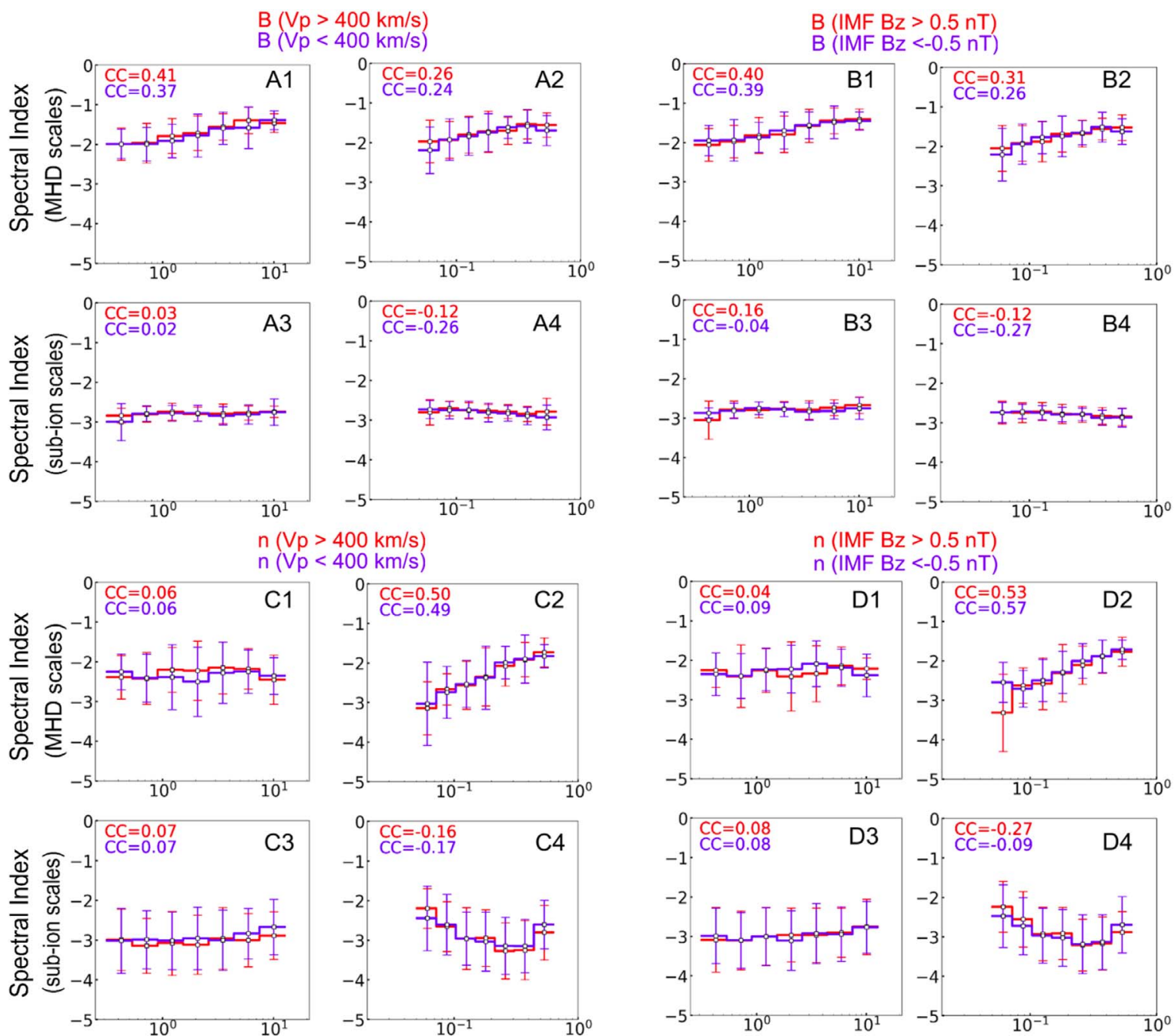

$V(V p>400 \mathrm{~km} / \mathrm{s})$
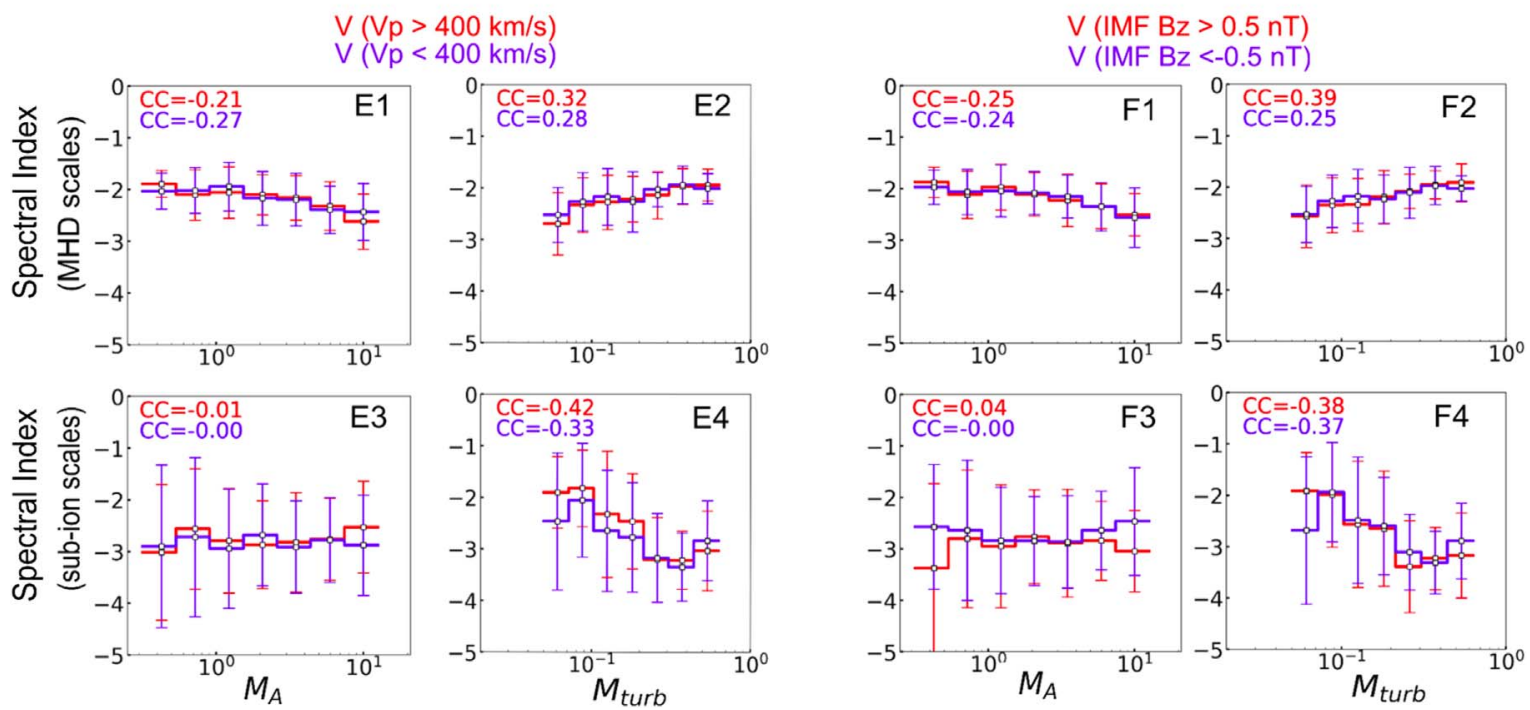

Figure 5. Comparisons of the relations between the turbulence spectral indices and $M_{\mathrm{A}}, M_{\mathrm{turb}}$ for different upstream solar wind conditions, such as the averaged $z$ component of interplanetary magnetic field (IMF $\left.B_{\mathrm{z}}\right)$ and the solar wind speed $\left(V_{\mathrm{p}}\right)$.

we study two aspects of the spatial evolution of magnetosheath turbulence at both MHD and sub-ion scales statistically.

From the subsolar region to the flanks, $M_{\mathrm{A}}$ increases. The spectral index of magnetic-field spectra at MHD scales changes from -2 for $M_{\mathrm{A}}<0.8$ to $-3 / 2$ for $M_{\mathrm{A}}>5$, presenting a positive correlation with $M_{\mathrm{A}}$. For the density spectra at MHD scales, the spectral index remains $\sim-7 / 3$ for different $M_{\mathrm{A}}$. The spectral index of velocity spectra at MHD scales changes from -2 for $M_{\mathrm{A}}<0.8$ to $-5 / 2$ for $M_{\mathrm{A}}>5$, presenting a negative correlation with $M_{\mathrm{A}}$. At sub-ion scales, we find no obvious 
relations between the spectral index and $M_{\mathrm{A}}$ for all the three types of spectra.

We also investigate the evolution of magnetosheath turbulence from the bow shock to the magnetopause. We use two parameters to represent the relative distance of the spacecraft from the bow shock or the magnetopause. One is the widely used fractional distance, $D_{\text {frac }}$. The other is the turbulent sonic Mach number, $M_{\text {turb }}$, which generally decreases from $>0.4$ in the bow shock vicinity to $<0.1$ near the magnetopause. At MHD scales, the magnetic-field, density, and velocity spectra steepen from the bow shock to the magnetopause, especially in the quasi-parallel magnetosheath. The mean value of spectral index for magnetic-field spectra changes from -1.46 in the vicinity of the bow shock to -1.94 in the vicinity of the magnetopause. For density and velocity spectra, the index changes from -1.87 to -2.44 and from -1.92 to -2.18 , respectively. At sub-ion scales, indistinct opposite trends of radial evolution are observed. All three types of spectra present a slight flattening from the bow shock to the magnetopause. Furthermore, the spectral indices are positively correlated with $M_{\text {turb }}$ at MHD scales, and negatively correlated with $M_{\text {turb }}$ at sub-ion scales. The break frequency of magneticfield and velocity spectra increases when approaching the magnetopause, from 0.24 to $0.33 \mathrm{~Hz}$ and from 0.32 to $0.43 \mathrm{~Hz}$, respectively. For the density spectra, the break frequency remains around $0.3 \mathrm{~Hz}$. Similar results, except for some minor differences, are found in the quasi-perpendicular magnetosheath. Our results might expand our knowledge on subsonic compressive MHD turbulence in the magnetosheath and contribute to understanding the transition from the subsonic to the supersonic regime.

In addition, we discuss the influences of upstream solar wind conditions, e.g., northward IMF $\left(B_{\mathrm{z}}>0.5 \mathrm{nT}\right)$ versus southward IMF $\left(B_{\mathrm{z}}<-0.5 \mathrm{nT}\right)$, fast wind $\left(V_{\mathrm{p}}>400 \mathrm{~km} \mathrm{~s}^{-1}\right)$ versus slow wind $\left(V_{\mathrm{p}}<400 \mathrm{~km} \mathrm{~s}^{-1}\right)$, on the spatial evolution of magnetosheath turbulence. We find no significant dependencies on the upstream parameters, suggesting that the spatial evolution of magnetosheath turbulence with a frequency greater than $0.02 \mathrm{~Hz}$ is largely independent of the averaged upstream solar wind conditions. These results are based on the hypothesis of a steady upstream solar wind. To what extent the penetrating small structures, such as plasma jets, especially behind a quasi-parallel shock (Hietala et al. 2009), could influence the magnetosheath turbulence properties still needs further investigation in the future.

The authors thank the MMS team for providing the FGM and FPI data at the MMS Science Data Center (https://lasp.colorado. $\mathrm{edu} / \mathrm{mms} / \mathrm{sdc} / \mathrm{public} /$ ), and NASA CDAWEB (https://cdaweb. sci.gsfc.nasa.gov/index.html/) for providing the OMNI data. Special thanks to Dr. B. B. Tang, Dr. W. Y. Li, and Prof. X. C. Guo for helpful discussions. This work was supported by Strategic Priority Research Program of Chinese Academy of Sciences grant No. XDA17010301, NNSFC grants 41874203, 41574169, 41574159, 41731070, Young Elite Scientists Sponsorship Program by CAST, 2016QNRC001, and grants from Chinese Academy of Sciences (QYZDJ-SSW-JSC028, XDA15052500). H.L. was also supported by the project of Civil Aerospace "13th Five Year Plan" Preliminary Research in Space Science (Project Name: Research on Important Scientific Issues of Heliospheric Boundary Exploration, Project No.: D020301), Youth Innovation Promotion Association of the Chinese Academy of Sciences and in part by the Specialized Research Fund for State Key Laboratories of China. D.V. is supported by the STFC Ernest Rutherford Fellowship ST/P003826/1 and STFC Consolidated Grant ST/S000240/1.

\section{ORCID iDs}

Hui Li (1) https://orcid.org/0000-0002-4839-4614

Daniel Verscharen (1D https://orcid.org/0000-0002-0497-1096

C. T. Russell (i) https://orcid.org/0000-0003-1639-8298

J. L. Burch (10 https://orcid.org/0000-0003-0452-8403

\section{References}

Alexandrova, O. 2008, NGeo, 15, 95

Alexandrova, O., Chen, C. H. K., Sorriso-Valvo, L., Horbury, T. S., \& Bale, S. D. 2013, SSRv, 178, 101

Alexandrova, O., Lacombe, C., \& Mangeney, A. 2008, AnGeo, 26, 3585

Alexandrova, O., Saur, J., Lacombe, C., et al. 2009, PhRvL, 103, 165003

Anderson, B. J., \& Fuselier, S. A. 1993, JGR, 98, 1461

Anderson, B. J., Fuselier, S. A., Gary, S. P., \& Denton, R. E. 1994, JGR, 99, 5877

Bale, S. D., Kellogg, P. J., Mozer, F. S., Horbury, T. S., \& Reme, H. 2005, PhRvL, 94, 215002

Banerjee, S., \& Galtier, S. 2013, PhRvE, 87, 013019

Breuillard, H., Matteini, L., Argall, M. R., et al. 2018, ApJ, 859, 127

Bruno, R., \& Carbone, V. 2013, LRSP, 10, 2

Bruno, R., Telloni, D., Sorriso-Valvo, L., et al. 2019, A\&A, 627, A96

Burch, J. L., Moore, T. E., Torbert, R. B., \& Giles, B. L. 2016, SSRv, 199, 5

Chao, J. K., Wu, D. J., Lin, C.-H., et al. 2002, in COSPAR Colloq. Ser. 12 , Space Weather Study Using Multipoint Techniques, ed. L.-H. Lyu (Amsterdam: Elsevier), 127

Chen, C. H. K., \& Boldyrev, S. 2017, ApJ, 842, 122

Chen, C. H. K., Leung, L., Boldyrev, S., Maruca, B. A., \& Bale, S. D. 2014, GeoRL, 41, 8081

Chhiber, R., Chasapis, A., Bandyopadhyay, R., et al. 2018, JGRA, 123, 9941

Czaykowska, A., Bauer, T. M., Treumann, R. A., \& Baumjohann, W. 2001, AnGeo, 19, 275

Dimmock, A. P., Nykyri, K., \& Pulkkinen, T. I. 2014, JGRA, 119, 6231

Fairfield, D. H. 1976, RvGeo, 14, 117

Galtier, S. 2006, JPIPh, 72, 721

Gary, S. P., Li, H., O’Rourke, S., \& Winske, D. 1998, JGR, 103, 14567

Goldstein, M. L., Wicks, R. T., Perri, S., \& Sahraoui, F. 2015, RSPTA, 373, 20140147

Gotoh, T., \& Kraichnan, R. H. 1993, PhFl, 5, 445

Guicking, L., Glassmeier, K.-H., Auster, H.-U., Narita, Y., \& Kleindienst, G. 2012, AnGeo, 30, 1271

Gurnett, D. A., Anderson, R. R., Tsurutani, B. T., et al. 1979, JGR, 84, 7043

Gutynska, O., Šimůnek, J., Šafránková, J., Němeček, Z., \& Přech, L. 2012, JGRA, 117, A04214

Hadid, L. Z., Sahraoui, F., Kiyani, K. H., et al. 2015, ApJL, 813, L29

Hellinger, P., \& Matsumoto, H. 2000, JGR, 105, 10519

Hietala, H., Laitinen, T. V., Andréeová, K., et al. 2009, PhRvL, 103, 245001

Howes, G. G., Klein, K. G., \& TenBarge, J. M. 2014, ApJ, 789, 106

Huang, S. Y., Hadid, L. Z., Sahraoui, F., Yuan, Z. G., \& Deng, X. H. 2017, ApJL, 836, L10

Huang, S. Y., Sahraoui, F., Deng, X. H., et al. 2014, ApJL, 789, L28

Huang, S. Y., Sahraoui, F., Yuan, Z. G., et al. 2018, ApJ, 861, 29

Iroshnikov, R. S. 1964, SvA, 7, 566

Karimabadi, H., Roytershteyn, V., Vu, H. X., et al. 2014, PhP1, 21, 062308

Kim, J., \& Ryu, D. 2005, ApJL, 630, L45

Klein, K. G., Howes, G. G., \& TenBarge, J. M. 2014, ApJL, 790, L20

Klein, L., Bruno, R., Bavassano, B., \& Rosenbauer, H. 1993, JGR, 98, 7837

Kolmogorov, A. N. 1941, DoSSR, 30, 299

Konstandin, L., Schmidt, W., Girichidis, P., et al. 2016, MNRAS, 460, 4483

Kowal, G., \& Lazarian, A. 2007, ApJL, 666, L69

Kowal, G., Lazarian, A., \& Beresnyak, A. 2007, ApJ, 658, 423

Kraichnan, R. H. 1965, PhFl, 8, 1385

Kritsuk, A. G., Norman, M. L., Padoan, P., \& Wagner, R. 2007, ApJ, 665, 416 Kunz, M. W., Schekochihin, A. A., \& Stone, J. M. 2014, PhRvL, 112, 205003 Lithwick, Y., \& Goldreich, P. 2001, ApJ, 562, 279

Lucek, E. A., Constantinescu, D., Goldstein, M. L., et al. 2005, SSRv, 118, 95 Lucek, E. A., Dunlop, M. W., Horbury, T. S., et al. 2001, AnGeo, 19, 1421 Macek, W. M., Krasińska, A., Silveira, M. V. D., et al. 2018, ApJL, 864, L29 
Macek, W. M., Wawrzaszek, A., \& Sibeck, D. G. 2015, JGRA, 120, 7466

Marsch, E., \& Tu, C.-Y. 1990, JGR, 95, 11945

Matteini, L., Alexandrova, O., Chen, C. H. K., et al. 2017, MNRAS, 466, 945

Matteini, L., Stansby, D., Horbury, T. S., \& Chen, C. H. K. 2018, ApJL, 869, L32

Omidi, N., O'Farrell, A., \& Krauss-Varban, D. 1994, AdSpR, 14, 45

Pollock, C., Moore, T., Jacques, A., et al. 2016, SSRv, 199, 331

Quest, K. B., \& Shapiro, V. D. 1996, JGR, 101, 24457

Rakhmanova, L., Riazantseva, M., Zastenker, G., et al. 2018a, JGRA, 123,5300

Rakhmanova, L. S., Riazantseva, M. O., \& Zastenker, G. N. 2015, CosRe, 53,331

Rakhmanova, L. S., Riazantseva, M. O., Zastenker, G. N., \& Verigin, M. I. 2018b, Ge\&Ae, 58, 718

Rakhmanova, L. S., Riazantseva, M. O., Zastenker, G. N., \& Yermolaev, Yu. I. 2017, JPIPh, 83, 705830204

Rezeau, L., \& Belmont, G. 2001, SSRv, 95, 427

Rezeau, L., Belmont, G., Cornilleau-Wehrlin, N., Reberac, F., \& Briand, C. 1999, GeoRL, 26, 651

Riazantseva, M. O., Rakhmanova, L. S., Zastenker, G. N., et al. 2017, Ge\&Ae, 57,1

Russell, C. T., Anderson, B. J., Baumjohann, W., et al. 2016, SSRv, 199, 189

Šafránková, J., Němeček, Z., Němec, F., et al. 2015, ApJ, 803, 107

Šafránková, J., Němeček, Z., Němec, F., et al. 2016, ApJ, 825, 121
Šafránková, J., Němeček, Z., Přech, L., \& Zastenker, G. N. 2013, PhRvL, 110, 025004

Sahraoui, F., Belmont, G., Rezeau, L., et al. 2006, PhRvL, 96, 075002

Sahraoui, F., Goldstein, M. L., Robert, P., \& Khotyaintsev, Y. V. 2009 PhRvL, 102, 231102

Sahraoui, F., Huang, S. Y., Belmont, G., et al. 2013, ApJ, 777, 15

Schekochihin, A. A., Cowley, S. C., Dorland, W., et al. 2009, ApJS, 182, 310

Schwartz, S. J., Burgess, D., \& Moses, J. J. 1996, AnGeo, 14, 1134

Shevyrev, N. N., Zastenker, G. N., \& Du, J. 2007, P\&SS, 55, 2330

Shevyrev, N. N., Zastenker, G. N., Eiges, P. E., \& Richardson, J. D. 2006 AdSpR, 37, 1516

Shue, J.-H., Chao, J. K., Fu, H. C., et al. 1997, JGR, 102, 9497

Southwood, D. J., \& Kivelson, M. G. 1993, JGR, 98, 9181

Teh, W.-L., \& Zenitani, S. 2019, ApJ, 885, 22

Verigin, M. I., Tátrallyay, M., Erdős, G., \& Kotova, G. A. 2006, AdSpR, 37,515

Verscharen, D., Chandran, B. D. G., Klein, K. G., \& Quataert, E. 2016, ApJ, 831,128

Vörös, Z., Yordanova, E., Graham, D. B., et al. 2019, JGRA, 124, 8551

White, T. G., Oliver, M. T., Mabey, P., et al. 2019, NatCo, 10, 1758

Yordanova, E., Vaivads, A., André, M., et al. 2008, PhRvL, 100, 205003

Zhao, J. S., Wang, T. Y., Dunlop, M. W., et al. 2019a, GeoRL, 46, 4545

Zhao, S. Q., Xiao, C. J., Wang, X. G., et al. 2019b, JGRA, 124, 10200

Zhu, X., He, J., Verscharen, D., \& Zhao, J. 2019, ApJ, 878, 48

Zimbardo, G., Greco, A., Sorriso-Valvo, L., et al. 2010, SSRv, 156, 89 